Relations industrielles

Industrial Relations

\title{
Smiling Down the Line: Info-Service Work in the Global \\ Economy, Par Bob Russell, Toronto : University of Toronto \\ Press, 2009, 326 p., ISBN 978-1-4426-0981-5
}

\section{Gilles Marcoux}

Volume 66, numéro 1, hiver 2011

URI : https://id.erudit.org/iderudit/1005114ar

DOI : https://doi.org/10.7202/1005114ar

Aller au sommaire du numéro

Éditeur(s)

Département des relations industrielles de l’Université Laval

ISSN

0034-379X (imprimé)

1703-8138 (numérique)

Découvrir la revue

Citer ce compte rendu

Marcoux, G. (2011). Compte rendu de [Smiling Down the Line: Info-Service Work in the Global Economy, Par Bob Russell, Toronto : University of Toronto Press, 2009, 326 p., ISBN 978-1-4426-0981-5]. Relations industrielles / Industrial

Relations, 66(1), 158-160. https://doi.org/10.7202/1005114ar

Tous droits réservés (C Département des relations industrielles de l’Université Laval, 2011
Ce document est protégé par la loi sur le droit d'auteur. L'utilisation des services d'Érudit (y compris la reproduction) est assujettie à sa politique d'utilisation que vous pouvez consulter en ligne.

https://apropos.erudit.org/fr/usagers/politique-dutilisation/ 
of nurses - such as reassuring patients and their families - as being too often attributed to 'innate' feminine traits and thus not financially rewarded. But even within this profession lies a class distinction - between high end positions that use technology and low end, high-touch jobs such as bathing patients. A gender distinction is added when the author turns to male nurses, who are often seen with more authority and benefit from assumptions about career development and leadership potential. When the author turns her attention to beauty parlours, she explains how women in these occupations emphasize professionalism while using their personalities to build and maintain relationships with clients.

In chapter 8, the author explores fast food and hotel workers to illustrate how gender and ethnicity intersect to create a suitability hierarchy for different positions. In fast food, the norms of masculinity for unskilled, working class young men are at odds with the behaviour expected in such occupations, putting them at a disadvantage. As for hotel workers, the focus shifts to ethnicity and how certain groups are associated with certain attributes, thus concentrating their presence in certain positions. The author then discusses the role of international employment agencies in the assembling of transnational labour forces and their racialized, gendered divisions.

One does not have to be an expert to understand McDowell's arguments, as the case studies and ethnographies she describes illustrate them well and render them accessible. In addition, the theoretical constructs she calls upon are well explained and permeate the whole book. Although her focus is on the UK, the comparisons she makes to the US and elsewhere are useful and show how most processes she describes are not unique to a single country. Overall, the book is insightful and could be recommended to students and experts alike.

\section{Carol-Anne Gauthier}

Université Laval

\section{Smiling Down the Line: Info-Service Work in the Global Economy}

Par Bob Russell, Toronto : University of Toronto Press, 2009, 326 p., ISBN 978-14426-0981-5

Le secteur des centres d'appels a connu, au cours des vingt dernières années, un essor considérable. Cette croissance a généré une multitude d'études portant sur ces milieux de travail et, bien souvent, plusieurs d'entre elles ont emprunté la perspective de la théorie du procès de travail (labour process theory) pour procéder à leurs analyses. Bien que cette démarche ait permis une meilleure compréhension de la réalité des centres d'appels en mettant en relief les enjeux relatifs au contrôle et à l'intensité du travail, Russell estime que ces études sont difficilement parvenues à mettre en évidence les perceptions des agents de service au sujet des situations expérimentées dans leur travail (p. 31). Pour pallier cette lacune, il propose d'inscrire son ouvrage dans la perspective d'une analyse matérialiste élargie (extended materialist analysis) s'appuyant sur plusieurs approches théoriques, en l'occurrence les théories de l'organisation, le courant de la théorie critique du management et celui de la mobilisation sociale.

L'ouvrage est divisé en neuf chapitres et il s'articule autour de trois questions directrices. D'abord, comment les agents de service travaillent-ils ou quel type d'organisation du travail applique-t-on dans les centres d'appels pour produire et livrer un service informationnel intangible et instantané ? Cette première question vise à analyser la thématique relative à la division technique du travail. Ensuite, où ces agents travaillent-ils, compte tenu notamment de la délocalisation de plusieurs centres d'appels dans des pays en développement ces dernières années ? Cette interrogation cherche à mettre en évidence les paramètres liés à la division sociale du travail dans ce secteur de l'économie. Enfin, quelles 
sont les implications des réponses fournies aux deux premières questions sur le plan de la gestion du personnel ? Cette dernière question oriente la réflexion autour des enjeux relatifs au managérialisme et à la gestion des ressources humaines dans les centres d'appels.

Après avoir présenté les trois études de cas sur lesquelles est basé l'ouvrage et après avoir décrit certains enjeux liés à l'occupation d'agent de service, Russell se penche sur la question de la division technique du travail. D'abord, au chapitre quatre, il effectue une analyse du procès de travail en centre d'appels et fait un examen des compétences requises chez les agents. Ensuite, au chapitre cinq, l'auteur élargit son analyse du procès de travail pour examiner les décisions managériales relatives aux technologies utilisées dans ces lieux de travail. II constate alors que les travailleurs dans ce secteur sont davantage qualifiés que ceux intégrés aux chaînes de montage sous l'ère fordiste, sans toutefois l'être autant que ceux évoluant actuellement dans le secteur de l'économie du savoir. De plus, son analyse l'amène à affirmer que nonobstant le fait que les agents ont rarement l'occasion d'influencer les décisions managériales sur le plan technologique, ils disposent toutefois d'un certain contrôle sur les manières d'utiliser ces technologies.

Par la suite, Russell se penche sur la question de la gestion du personnel ainsi que sur celle des réactions des employés à l'égard de celle-ci. Au chapitre six, il met alors en évidence de quelle façon les gestionnaires contemporains, en articulant leur argumentation autour du concept de managérialisme, s'intéressent aux questions de culture, d'identité et d'engagement dans leurs organisations. De plus, il relate les opinions et les réactions des employés à l'égard des pratiques managériales relatives au managérialisme et il en profite, au chapitre huit, pour aborder la question de leur résistance et celle de la présence syndi- cale dans ces lieux de travail. Le principal constat de l'auteur se rapporte au fait que le managérialisme atteint difficilement ses objectifs liés à l'accroissement de l'identité et de l'engagement des employés à l'égard de l'organisation. En fait, les mécanismes de contrôle relatifs à l'application du managérialisme génèrent souvent des problèmes de confiance de la part des salariés à l'égard de la direction, de sorte que peuvent être constatées diverses formes de résistance incluant la syndicalisation.

Enfin, l'auteur porte son attention sur la division sociale du travail dans les centres d'appels en ayant recours au phénomène lié à leur délocalisation dans les pays en développement. À cet égard, Russell s'interroge sur les différences et les similarités relatives au travail en centre d'appels qu'il est possible d'identifier entre des sites australiens et des sites indiens. Le premier constat de l'auteur concerne le fait que le travail, en termes d'intensité et de fluidité des opérations, semble relativement similaire entre ces sites. Ainsi, le phénomène de mondialisation entraînerait, à tout le moins dans ce secteur d'activités, une externalisation du procès de travail. Le second constat se rapporte au fait que les exigences requises en termes de compétences seraient comparables entre ces régions du monde de sorte qu'il ne semble pas y avoir d'exportation du travail déqualifié vers les centres d'appels du Sud (p. 232-233).

La principale force de cet ouvrage réside dans le fait qu'en ayant recours à diverses théories pour approfondir le courant sur le procès de travail, Russell est parvenu à élargir l'angle analytique habituel des études portant sur les centres d'appels. Ainsi, il a été en mesure de démontrer comment le procès de travail appliqué dans la production de services informationnels intangibles et instantanés a modifié jusqu'à un certain point la conception du travail dans le secteur tertiaire en termes de divisions technique et sociale du travail et en termes de gestion des salariés. De plus, 
la démarche de l'auteur a permis d'identifier les angles d'analyse pertinents que devraient adopter les recherches futures sur les centres d'appels. Toutefois, bien que Russell exprime, au début de son ouvrage, une volonté à mettre davantage en évidence les perceptions des employés à l'égard de leur expérience de travail, et $c e$, en misant sur une compréhension de leur interprétation situationnelle, il semble que la portée de son analyse sur ce plan ne dépasse pas vraiment ce qui a déjà été réalisé dans le passé. En fait, l'analyse de Russell tend globalement, elle aussi, à répondre à la question des facteurs qui amènent les agents à être insatisfaits de leurs conditions de travail. Dans le contexte où il demeure évident qu'un nombre d'individus travaillant en centres d'appels y demeurent pendant une période de temps non négligeable (voir Gilles Marcoux, « Engagement organisationnel et distanciation sociale : analyse interprétative de la situation expérimentée par les agents de service en centre d'appel ", thèse de doctorat, Département des relations industrielles, Université Laval, Québec, 2007) ou qu'ils y font même carrière, il importe, à notre avis, d'élargir la portée des études portant sur ces milieux de travail. Si on dépasse les analyses relatives par exemple à la rémunération, à la résistance ou aux faibles possibilités d'accomplissement dans ce genre de travail, ne serait-il pas possible de se demander pourquoi plusieurs individus demeurent en emploi dans les centres d'appels ? Certains éléments de leur travail peuvent-ils donner un sens, si minime soitil, à leurs tâches quotidiennes et les inciter à rester dans ce genre d'emploi ? Dans le passé, certaines études ont démontré que les agents échappaient aux désagréments de leur travail (p. 170) en trouvant satisfaction dans la relation qu'ils expérimentent avec la clientèle. Or, puisque ce type de relation se déroule à distance et qu'elle est souvent porteuse d'agressivité de la part de la clientèle envers les agents, la rela- tion avec le client ne peut expliquer, à elle seule, les vecteurs de sens lié à ce genre de travail.

Bref, Russell lance une piste intéressante lorsqu'il traite de la question de l'identité au chapitre six. Toutefois, il I'aborde surtout en ayant recours à l'identité organisationnelle. Compte tenu des limites de cette forme d'identité et de celle liée à la clientèle, il serait certainement pertinent d'élargir l'angle analytique pour explorer I'idée que les agents de service ont certainement une identité envers leur rôle et leur métier qui, peut-être, les aide à persévérer dans ce genre de travail. Ainsi, la piste de l'identité semble prometteuse pour les futures recherches portant sur les centres d'appels puisqu'elle permettrait de dépasser les analyses habituelles s'articulant autour de la question de la résistance pour mettre davantage en valeur les compétences requises, probablement sociales, pour non seulement effectuer ce travail efficacement mais aussi pour y persévérer et lui donner un certain sens.

\section{Gilles Marcoux}

Université de Moncton

\section{Questions sociales : analyses anglo-saxonnes - Socialement incorrect?}

Par Julien Damon, Paris: Presses

Universitaires de France, 2009, 240 p., ISBN

978-2-13-957918-9

Alors que les décideurs politiques et les acteurs sociaux se débattent sur la direction et sur les moyens de relancer les économies et de préserver la protection sociale à la suite de la crise financière et économique des années 2008-2009, cet ouvrage porte un éclairage fort utile sur une trentaine d'ouvrages d'analyse et de critique anglo-saxons des quinze dernières années concernant les orientations de l'État-Providence, des politiques sociales et des divers objectifs qu'elles poursuivent ou devraient poursuivre et sur les moyens de mise en œuvre de 